\title{
COIL emission of a modified negative branch confocal unstable resonator
}

\author{
Carsten Pargmann, ${ }^{1, \star}$ Thomas Hall, ${ }^{2}$ Frank Duschek, ${ }^{1}$ Karin Maria Grünewald, ${ }^{1}$ and Jürgen Handke ${ }^{1}$ \\ ${ }^{1}$ German Aerospace Center (DLR), Institute of Technical Physics, Langer Grund, D-74239 Hardthausen, Germany \\ ${ }^{2}$ German Aerospace Center (DLR), Institute of Technical Physics, Pfaffenwaldring 38-40, D-70569 Stuttgart, Germany
}

${ }^{*}$ Corresponding author: carsten.pargmann@dlr.de

Received 20 August 2007; revised 18 September 2007; accepted 21 September 2007; posted 21 September 2007 (Doc. ID 86679); published 29 October 2007

\begin{abstract}
A modified negative branch confocal unstable resonator (MNBUR) was coupled to the chemical oxygeniodine laser (COIL) device of the German Aerospace Center. It consists of two spherical mirrors and a rectangular scraper for power extraction. Experimentally measured distributions of the near- and farfield intensities and the near-field phase were found in close agreement to numerical calculations. The extracted power came up to $\sim 90 \%$ of the power as expected for a stable resonator coupled to the same volume of the active medium. The output power revealed a considerable insensitivity towards tilts of the resonator mirrors and the ideal arrangement of the scraper was found to be straightforward by monitoring the near-field distributions of intensity and phase. The beam quality achieved with the MNBUR of an extremely low magnification of only 1.04 was rather poor but nevertheless in accordance with theory. The demonstrated consistency between theory and experiment makes the MNBUR an attractive candidate for lasers that allow for higher magnification. In particular, it promises high brilliance in application to $100 \mathrm{~kW}$ class COIL devices, superior to the conventional negative branch confocal unstable resonator. (C) 2007 Optical Society of America

OCIS codes: $140.1550,140.3410,140.4780,230.5750$.
\end{abstract}

\section{Introduction}

For a typical chemical oxygen-iodine laser (COIL) with low gain and a large rectangular cross section, it is difficult to design a resonator that produces a timeindependent mode and a respectable far-field intensity distribution. The deployment of COIL as a tactical system imposes additional requirements on the resonator concept, such as compactness and mechanical stability.

A stable resonator with a high Fresnel number results in a multimode operation with poor beam quality. For unstable resonators with low gain, the total coupling loss has to be small [1]. The result is a far-field intensity distribution with a lot of structure and very small peak power. Hence, a conventional unstable resonator [2] is not well-suited for this type of laser. An unstable ring resonator with $90^{\circ}$ beam rotation $[3,4]$ has been shown to have good beam quality and align-

0003-6935/07/317751-06\$15.00/0

(C) 2007 Optical Society of America ment insensitivity, but the experimental setup is rather complicated. Different types of stable-unstable resonators [5-8] were used to improve the beam quality, but the disadvantage of such a hybrid resonator is its stable part. With increasing mirror dimensions, the problems of conventional stable resonators also occur in the stable direction of hybrid resonators. Furthermore the cylindrical mirrors are of a special design and are therefore expensive.

An off-axis hybrid resonator coupled to a $10 \mathrm{~kW}$ class COIL may extract near diffraction limited power output [9]. At much higher power levels $(\sim 1$ MW) the conventional unstable-resonator-type has to be favored, because the low gain is partially compensated by a larger volume of the active medium. Therefore, the amplification is increased and higher values of magnification are suitable. The modified negative branch confocal unstable resonator is a promising candidate to be the missing link for lasers with intermediate output power levels. In an earlier publication [10] the principle of the modified negative branch confocal unstable resonator (MNBUR) was 
explained theoretically using spherical geometry. Meanwhile, verification tests of the MNBUR were performed for the first time, to the best of our knowledge, and the resonator design proves to be easily adaptable to the rectangular geometry of the laser.

\section{Resonator Design}

The mirrors of this modified unstable resonator form an off-axis negative branch confocal resonator. The assembly is shown in Fig. 1 [back mirror (BM) and output mirror $(\mathrm{OM})]$. Two classical spherical mirrors were applied, making the setup simple and cost effective. The output coupling of the power and the adaptation to the rectangular geometry is not done by the OM, but is realized by a scraper. The manufacturing of the scraper is straightforward.

Figure 2(a) shows the resonator in the $x-z$ plane (the $z$ direction coincides with the optical axis of the resonator). The cross sections of the mirrors and the scraper in Fig. 2(b) are perpendicular to the $z$ plane. The focal points of the BM and OM coalesce between the mirrors. Nevertheless, an intracavity breakdown should not be a problem, because the common focal point can be positioned outside the gain medium range. Due to the rectangular cross section of the active medium and the shape of the scraper only parts of the spherical mirrors are hit by the radiation field. The relations between the dimensions on the mirrors are defined by $a_{2}=M a_{1}$ and $a_{3}=M a_{2}$ $=M^{2} a_{1} ; b_{2}=M b_{1}$ and $b_{3}=M b_{2}=M^{2} b_{1}$, respectively.
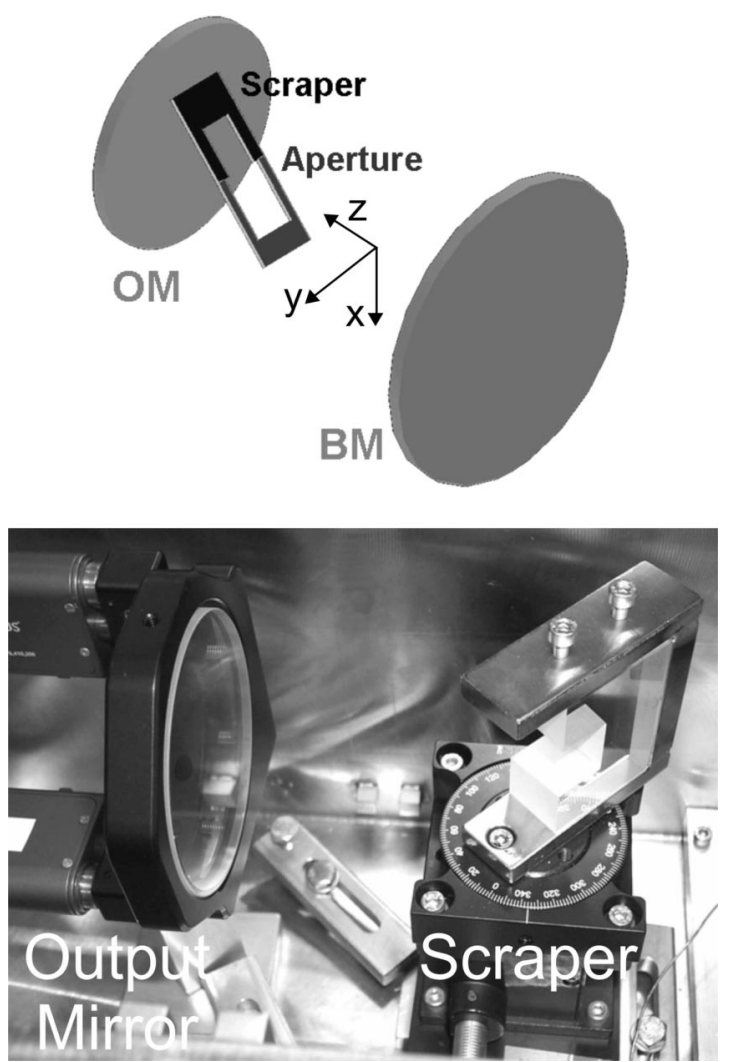

Fig. 1. Sketch and photograph of the MNBUR showing the output mirror $(\mathrm{OM})$, the back mirror $(\mathrm{BM})$, and the scraper.
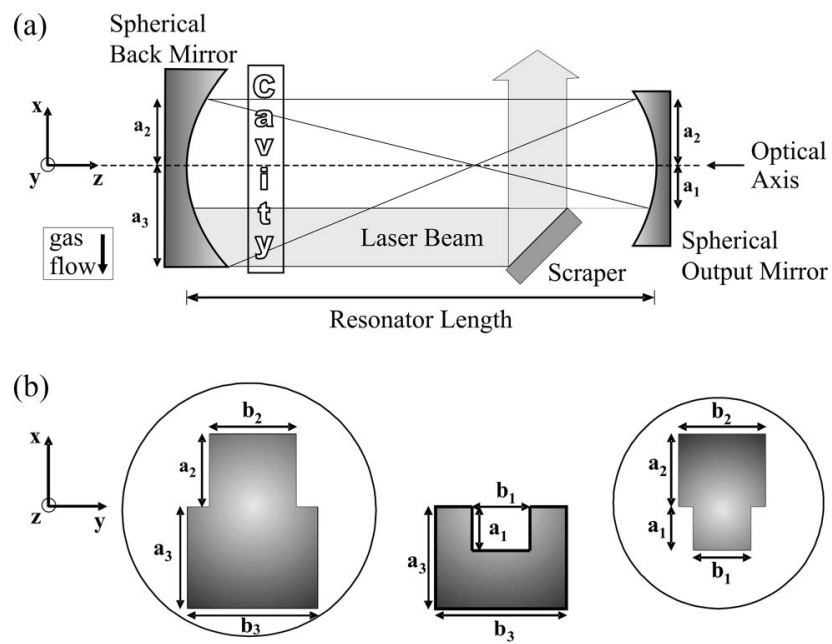

Back Mirror BM

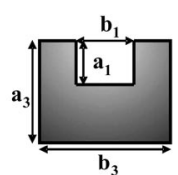

Scraper

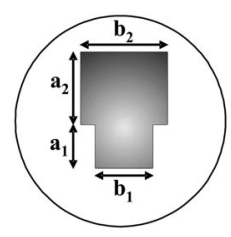

Output Mirror OM
Fig. 2. (a) Arrangement of a scraper in the MNBUR, and (b) the corresponding areas of the resonator mirrors and the scraper exposed to the radiation field.

The magnification $M$, i.e., the ratio of the radii of curvature of the $\mathrm{BM}\left(R_{\mathrm{BM}}\right)$ and the $\mathrm{OM}\left(R_{\mathrm{OM}}\right)$, $M=R_{\mathrm{BM}} / R_{\mathrm{OM}}$, determines the output coupling of the unstable resonator. The geometrical output coupling fraction $T$ of the modified off-axis negative branch confocal unstable resonator is given by $T=1-$ $1 / M^{2}$ and corresponds to the $T$ of the conventional negative branch confocal unstable resonator.

The scraper is positioned near the $\mathrm{OM}$ and is a plane mirror with a rectangular gap of edge lengths $a_{1}$ and $b_{1}$ in the $x$ and $y$ directions, respectively. The real lengths of the gap have to be adapted according to the angle between the scraper and the optical axis. The special shaping of the scraper together with the negative branch confocal unstable resonator configuration yields a laser output beam in the shape of a rectangular bracket, "[". In contrast to the conventional resonator with a rectangular and/or circularframe-shaped output beam, the cross-sectional area of the [ shape is broader and thereby more compact and generates a lower structured far-field distribution, but the near-field symmetry disappears with respect to the $y$ axis.

The centers of the curvature of the BM and OM are located on the optical axis. For proper alignment, the two caps of the [-shaped scraper have to lie on a straight line that intersects the optical axis perpendicularly.

\section{Numerical Computations}

The theory is based on the integral equation of the Fresnel-Kirchhoff formulation of Huygens principle [11]. The calculations describe a passive resonator, which is a resonator without consideration of gain properties. The implementation of the gain medium in the calculation should not provide significant new insights, because the measured small-signal gain of the German Aerospace Center (DLR)-COIL [12] is quite homogeneous over the whole gain region. 


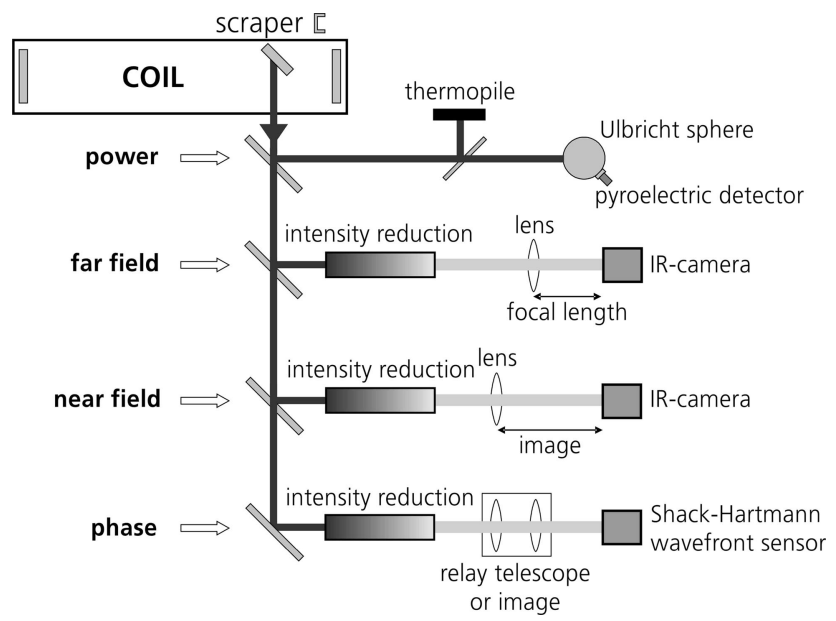

Fig. 3. Experimental setup.

Therefore, in this case, gain calculations are only required for the fine-tuning of the resonator and for special detailed features in intensity and phase distribution. The well-known Fox-Li method [13] is used to solve numerically the integral equation.

\section{Experimental Setup}

For the first experimental test about the feasibility of the MNBUR, and in order to verify the validity of the numerical computations, experimental setups have been built up to measure the intensity and phase distributions of the far and the near field of the laser beam. These intensity and phase distributions are acquired by a CCD-camera (Spiricon, COHU4812 or SCOR20). The intensity of the laser beam is attenuated by making use of reflections at wedge-shaped beam splitters. A scheme of the experimental setup is shown in Fig. 3. The power output is measured by two fast detectors coupled to an integrating sphere and a power meter for calibration.

The intensity distribution of the far field is obtained by placing the camera in the focus of a lens. The used focal length is $1480 \mathrm{~mm}$. The position of the

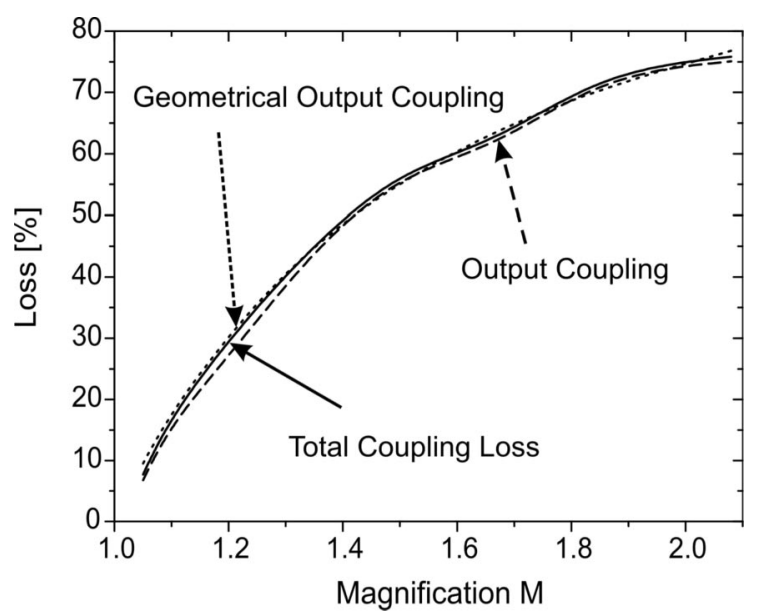

Fig. 4. Total coupling loss and output coupling of MNBUR versus magnification $M$.

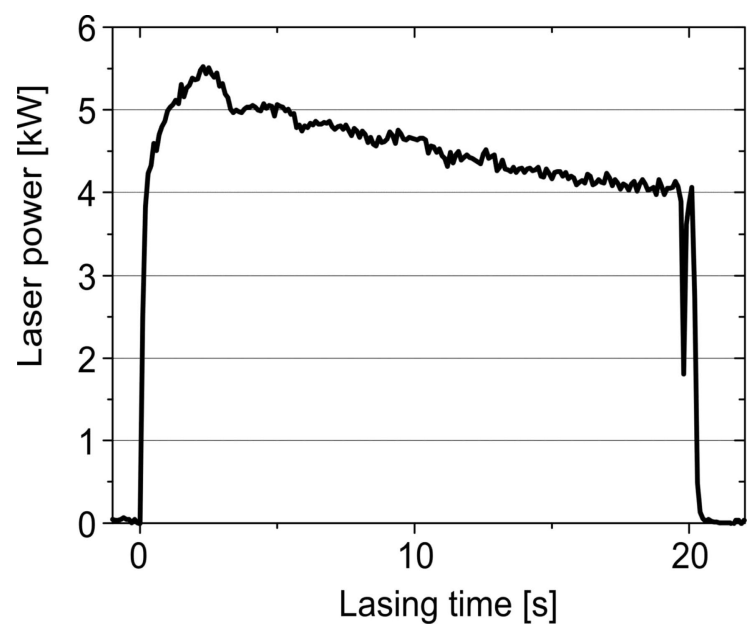

Fig. 5. Laser power extracted by MNBUR.

lens is kept close to the scraper. The intensity and the phase distribution of the near field are measured by imaging the field distribution at the scraper position onto the respective camera. The intensity of the near field is maintained in the image, but not the phase. This fact is taken into account when comparing to the numerical calculations. Subsequent phase measure-
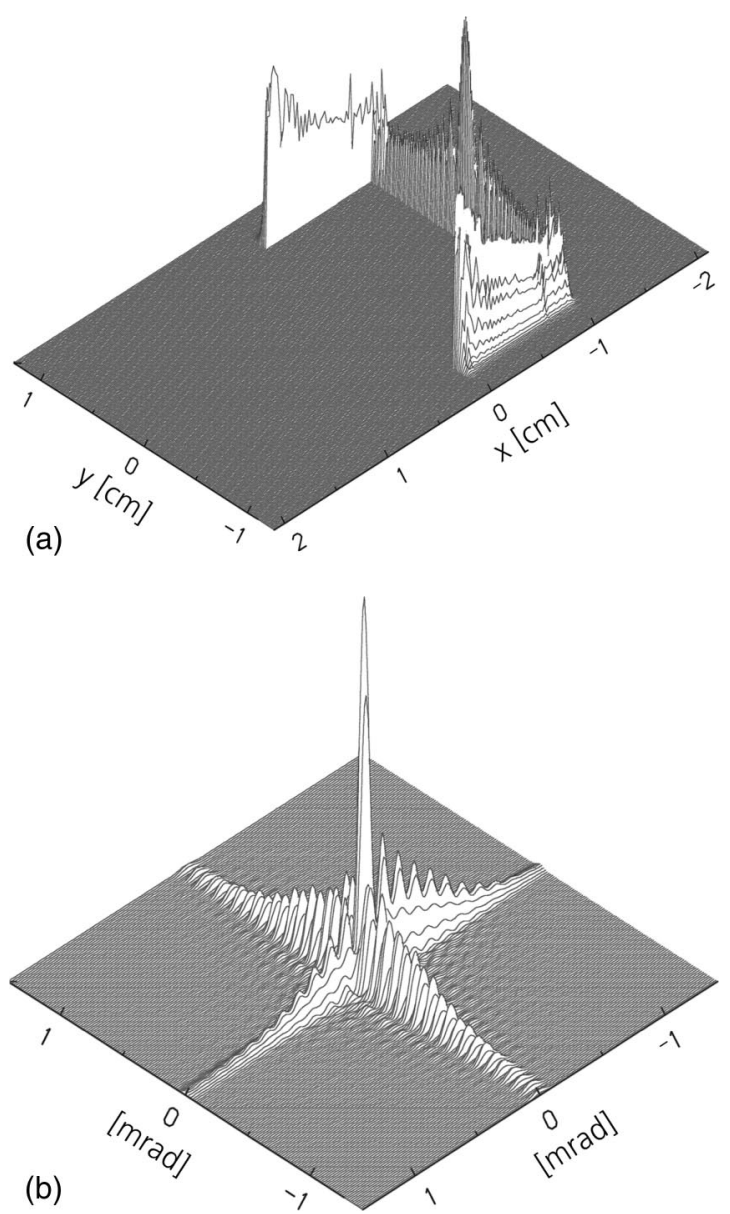

Fig. 6. Calculated (a) near- and (b) far-field intensity distributions of the optimal MNBUR. 
ments are done by using a relay telescope for imaging.

To get the phase distribution from an image, an array of microlenses is positioned in front of the CCD chip. The principle of calculating the phase distribution by measuring the spot positions was first introduced by Hartmann [14] and was later improved by the implementation of microlenses by Shack and Platt [15]. Concerning our sensor the microlenses built up an array of only $20 \times 16$ spots. To achieve a reasonable spatial resolution, only a part of the total emitting area of the MNBUR is imaged to the camera. Therefore the total image is obtained during several laser shots. Another shortcoming of the phase measurements arises if there is a lack of a plane reference wave to acquire the reference grid. To overcome this disadvantage the symmetry in the $y$ direction is used for the evaluation of the measured distributions, but the tilt in the $x$ direction had to be approximated according to a comparison with the numerical results. Nevertheless, with this method the obtained numerical and experimental trends in phase distribution can be compared.

\section{Experimental and Numerical Results}

The above-described resonator is coupled to the DLRCOIL device [16]. In Fig. 4 the total coupling loss and the output coupling of the MNBUR with a [-shape output beam are plotted versus the magnification $M$. The difference between the total coupling loss and the output coupling describes the diffraction loss of the resonator. For a first experimental test a low magnification of $M=1.04$ was designed to ensure that the resonator overcomes the threshold, at possibly unex-

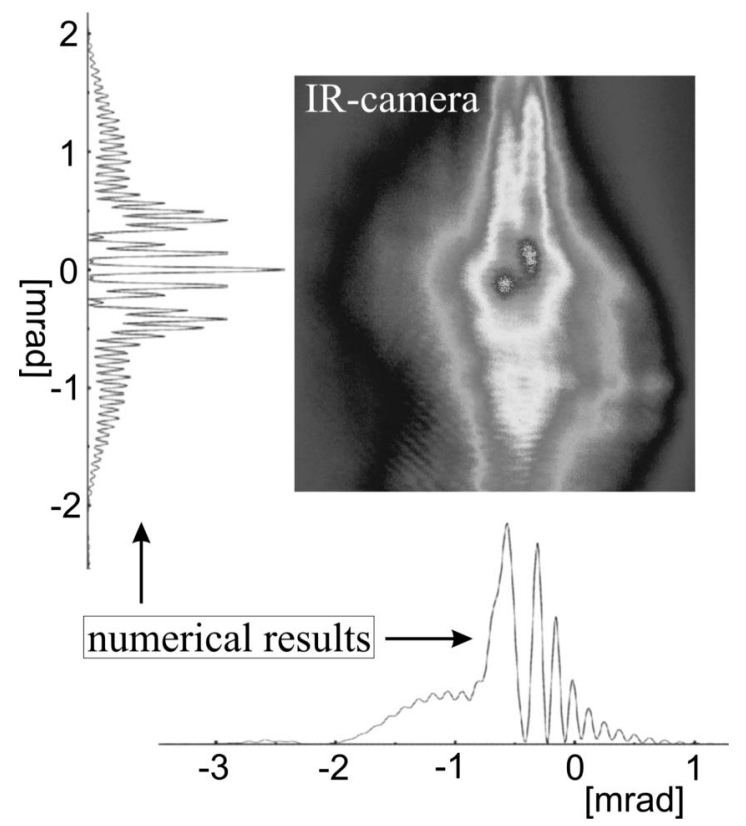

Fig. 7. Numerical and experimental far-field intensity distribution of a MNBUR with a slightly reduced resonator length in the focus of a lens. The numerical results are cuts through the center of the distribution. pected diffraction losses, too. The numerical calculation of the optimal resonator yields a total coupling loss of $5.6 \%$ and an output coupling of $5.4 \%$. The exploited gain medium is $19.5 \mathrm{~mm}$ in height and $21.2 \mathrm{~mm}$ in flow direction. The gain length amounts to $200 \mathrm{~mm}$ in the direction of the optical axis. From these data and from experimental results [16] a maximal laser output power of $6.3 \mathrm{~kW}$ is expected. In Fig. 5 the achieved laser power coupled out by the MNBUR is plotted. The laser power achieves a maximum value of $\sim 5.5 \mathrm{~kW}$; the power decrease with time is due to the batch mode operation of the COIL device with its rotating disk oxygen generator. The experimentally obtained output power is close to the predicted maximum value.

The calculated near- and far-field intensity distributions are shown in Fig. 6 . Because of the very small magnification, the central peak in the far field only contains a small amount of the total power and the distribution shows a lot of structure. According to the manufacturer's instructions the delivered resonator mirrors admit a magnification of $M=1.04$ with a radius of curvature of $1949 \mathrm{~mm}$ for the $\mathrm{OM}$ and of $2030 \mathrm{~mm}$ for the BM. Mirrors and scraper are high reflectivity coated for the COIL wavelength. Experimentally it was not possible to attain a far-field intensity distribution as narrow as assumed for the confocal configuration. Obviously the manufacturer's
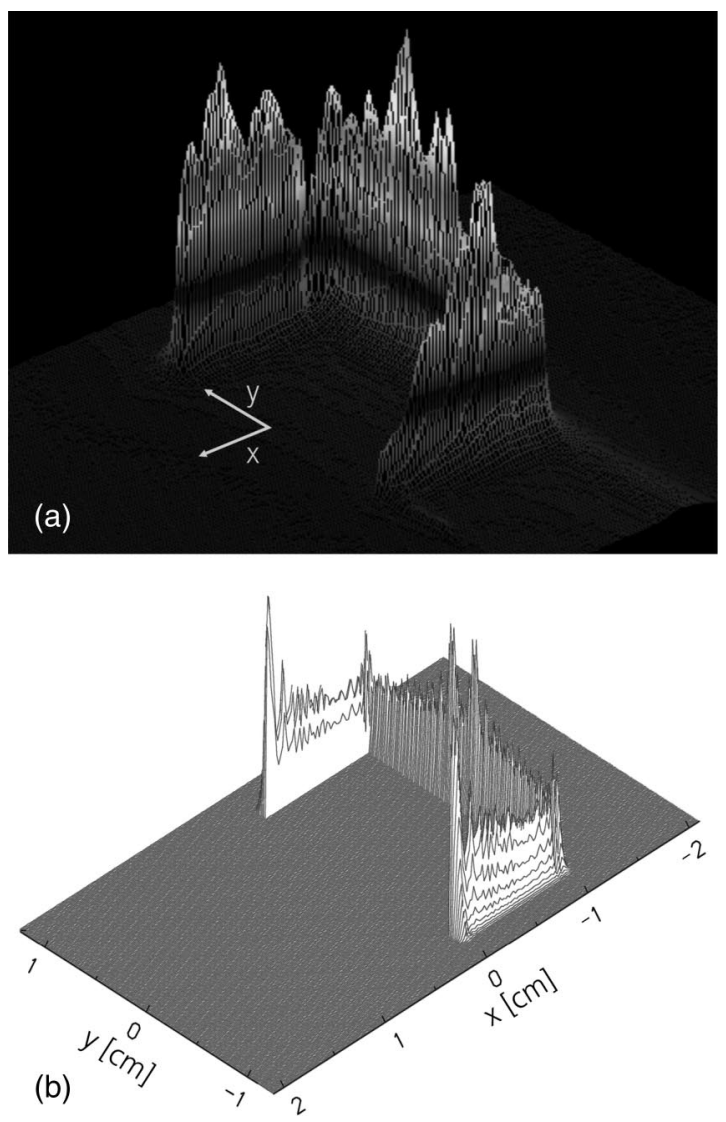

Fig. 8. (a) Measured and (b) calculated near-field intensity distribution of the confocal MNBUR. The calculation is performed with the assumption of a slightly reduced resonator length. 
instructions of the resonator mirrors are not exact and/or there is a little deviation from the spherical contour of the resonator mirrors. Because the difference between the radii of curvature is quite small, very small deviations from the specified mirror curvatures devastate the far field, as can be reproduced by numerical calculations. Experimentally the best results are derived from a resonator with a slightly reduced length. At this reduced resonator length, a correlation between the numerical and experimental results is shown in Fig. 7. For a better contrast the image of the IR camera is a black and white illustration of a color image. So apart from the ripples the intensity rises from the outer edge up to the two central peaks. The size of the far field, the slight asymmetry, and the frequency of the ripples basically coincide. The measured distribution shows somewhat less substructures, mainly due to integration in time.

The measured intensity of the near field is given in Fig. 8(a). It does not reveal such a distinct central peak as shown in the theoretical result for a confocal configuration (Fig. 6). In Fig. 8(b) a calculation of the near field with a slightly reduced resonator length is shown. The central peak is replaced by less pronounced structures and the calculation again comes close to the measured result. Irrespective of the as-
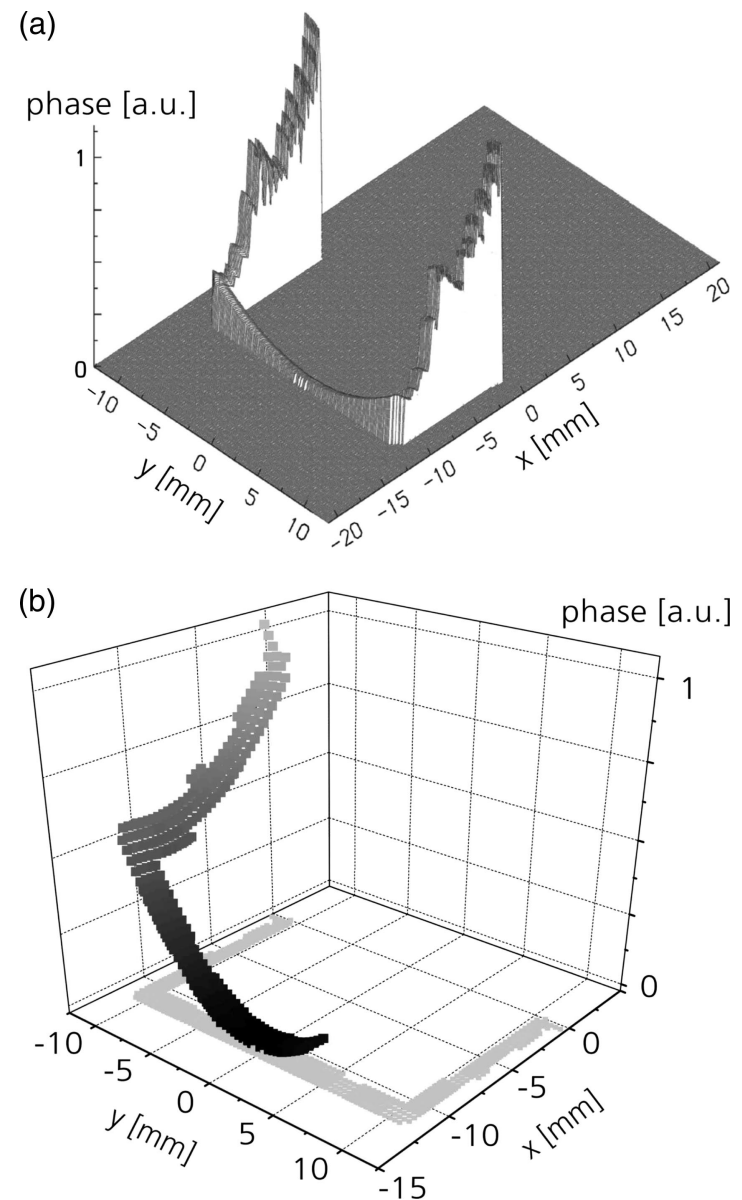

Fig. 9. (a) Calculated and (b) measured phase distribution of the near field behind a 1:1 image. sumed deviation from the confocality another reason for this pronounced peak in Fig. 6 can be given by the negligence of the gain of the active medium within the numerical calculations.

Figure 9 shows a comparison between the numerical and the experimental result of the phase distribution in the near field. Because of symmetry only half of the distribution has been measured. The obtained trends are very similar to each other. Both reveal a minimum at the center, and the slopes of the curves coincide. The phase distribution at the calculated outer wings is somewhat more irregular, but the measurements exhibit a much less spatial resolution than the calculations and may also be smoothened by fluctuations in time.

An advantage of the MNBUR compared to hybrid resonators is the greater insensitivity of the output power towards tilts of the resonator mirrors. Experimentally a tilt of the OM of $500 \mu \mathrm{rad}$ reduces the output power by less than 5\%. As mentioned before, the position of the scraper should also conform to an optimal condition. Moving the scraper $3 \mathrm{~mm}$ in any direction out of its optimum position reduces the output power by less than $10 \%$, but the intensity distribution of the near field changes. Figure 10 shows images of the calculated and measured intensity distributions together with phase measurements of the near field. The phases are measured at the lower right corner of the [ shape of the near field and are enlarged by a factor of 1.64 . The part of interest in the numerical calculations is marked by an oval. Moving the scraper horizontally out of the cavity leads to an evolution of substructures at the ends of the [ shape. Since the mirrors are larger than the scraper, additional area for the radiation field is released.

(a)

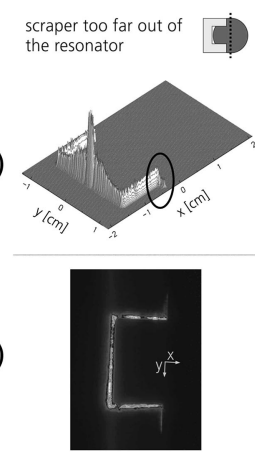

(c)

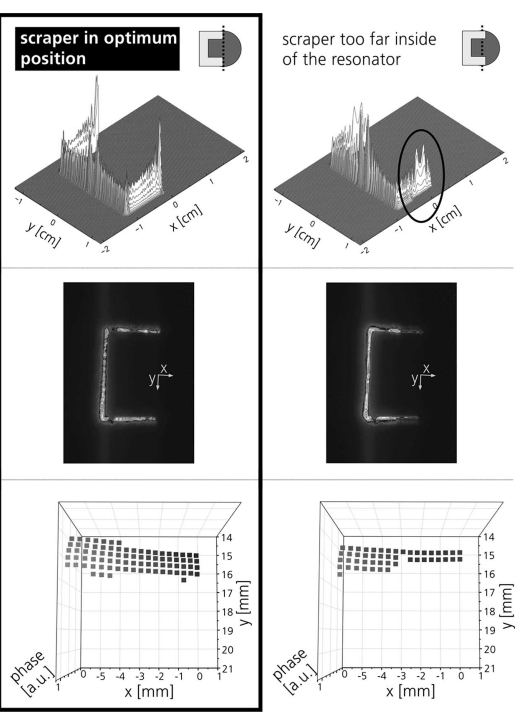

Fig. 10. Changes of the (a) calculated intensity distribution, (b) the measured intensity distribution, and (c) the measured phase distribution of the near field by moving the scraper horizontally in the cavity. In (a) and (b) the maximum scraper displacement is $3 \mathrm{~mm}$ and in (c) it is $1.5 \mathrm{~mm}$. The phase measurements show only the lower right corner of the [ shape. 
Thereby, two side wings appear. When the scraper position is too far inside of the cavity the width of the ends is reduced because parts of the nominal radiation field are shaded by the scraper. A vertical movement of the scraper leads to an asymmetric evolution of the substructures. The substructures are shown in the measurements as well as in the numerical computations and underline the good coincidence between both. Observations of the intensity distribution of the near field can help to automatically correct the position of the scraper if necessary. The far-field distributions are less affected by small deviations from the optimum position of the scraper.

\section{Conclusion}

A modified negative branch confocal unstable resonator (MNBUR) with a very low magnification was coupled to a chemical oxygen-iodine laser device. The output power achieved $\sim 90 \%$ of the predicted value. Intensity and phase measurements of the near and far field have been performed and compared with numerical calculations. Theoretical predictions and experimental results are in good agreement. The deviations of measured data from the calculation can be assigned to manufacturing imperfections, which are avoidable especially for higher magnification numbers. Even the evolution of substructures in the intensity distribution of the near field when the scraper position is changed coincides with the calculations. On the basis of this demonstration it is assumed, that the far field of a MNBUR with larger magnification and therefore resonator mirrors, which offer a larger difference in their radii of curvature, conforms to the numerical results, too. This will result in a much better concentration of the laser power into the central peak of the far field and will make the MNBUR a good choice for a laser of the $100 \mathrm{~kW}$ class superior to the conventional negative branch confocal unstable resonator.

The authors are grateful to G. Spindler for helpful technical discussions.

\section{References}

1. A. E. Siegman, "Unstable optical resonators for laser application," Proc. IEEE 53, 277-287 (1965).

2. A. E. Siegman, "Unstable optical resonators," Appl. Opt. 13, 353-367 (1974).

3. A. H. Paxton and W. P. Latham, Jr., "Unstable resonators with 90 beam rotation," Appl. Opt. 25, 2939-2946 (1986).

4. Y. Bin, B. Yang, F. Sang, D. Zhou, L. Dou, and Q. Zhuang, "Experimental investigations of an unstable ring resonator with 90-deg beam rotation for a chemical oxygen iodine laser," Appl. Opt. 38, 3249-3252 (1999).

5. O. L. Bourne and P. E. Dyer, "A novel stable-unstable resonator for beam control of rare-gas halide lasers," Opt. Commun. 31, 193-196 (1979).

6. N. Hodgson, T. Haase, and H. Weber, "Improved resonator design for rod lasers and slab lasers," Proc. SPIE 1277, 70-84 (1990).

7. K. Kuba, T. Yamamoto, and S. Yagi, "Improvement of slablaser beam divergence by using an off-axis unstable-stable resonator," Opt. Lett. 15, 121-123 (1990).

8. T. Hall, "Numerical studies on hybrid resonators for a mediumsized chemical oxygen iodine laser," Opt. Eng. 44, 114201 (2005).

9. J. Handke, W. O. Schall, T. Hall, F. Duschek, and K. M. Grünewald, "Chemical oxygen-iodine laser power generation with an off-axis hybrid resonator," Appl. Opt. 45, 3831-3838 (2006).

10. T. Hall, F. Duschek, K. M. Grünewald, and J. Handke, "Modified negative branch confocal unstable resonator," Appl. Opt. 45, 8777-8780 (2006).

11. J. W. Goodman, Introduction to Fourier Optics (McGraw-Hill, 1968).

12. K. M. Grünewald, J. Handke, and F. Duschek, "Small signal gain and temperature profiles in supersonic COIL," Proc. SPIE 4184, 75-78 (2000).

13. A. G. Fox and T. Li, "Resonant modes in a maser interferometer," Bell Syst. Tech. J. 40, 453-488 (1961).

14. J. Hartmann, "Bemerkungen über den Bau und die Justirung von Spektrographen," Z. Instrum. 20, 2-27 (1900).

15. R. V. Shack and B. C. Platt, "Production and use of a lenticular Hartmann screen," J. Opt. Soc. Am. 61, 656-660 (1971).

16. J. Handke, K. M. Grünewald, and W. O. Schall, "Power extraction in investigations for a 10-kW-class supersonic COIL," Proc. SPIE 3574, 309-314 (1998). 